\title{
Radio-frequency plasma cleaning for mitigation of high-power microwave-pulse shortening in a coaxial gyrotron
}

\author{
William E. Cohen, ${ }^{\text {a) }}$ Ronald M. Gilgenbach, ${ }^{\text {b) }}$ Reginald L. Jaynes, Christopher W. Peters, \\ Mike R. Lopez, Y. Y. Lau, Scott A. Anderson, and Mary L. Brake \\ Intense Energy Beam Interaction Laboratory, Nuclear Engineering and Radiological Sciences Department, \\ University of Michigan, Ann Arbor, Michigan 48109-2104 \\ Thomas A. Spencer \\ Air Force Research Laboratory-Phillips Research Laboratory, Kirtland AFB, New Mexico 87117-5776
}

(Received 31 August 2000; accepted for publication 11 October 2000)

Results are reported demonstrating that radio-frequency (rf) plasma cleaning is an effective technique for mitigating microwave-pulse shortening (i.e., lengthening the pulse) in a multimegawatt, large-orbit, coaxial gyrotron. Cleaning plasmas were generated by $50 \mathrm{~W}$ of rf power at $13.56 \mathrm{MHz}$ in nitrogen fill gas in the pressure range 15-25 mTorr. Improvements in the averaged microwave energy output of this high-power-microwave device ranged from $15 \%$ to $245 \%$ for different initial conditions and cleaning protocols. The mechanism for this improvement is believed to be rf plasma sputtering of excess water vapor from the cavity/waveguide and subsequent removal of the contaminant by cryogenic vacuum pumps. (C) 2000 American Institute of Physics.

[S0003-6951(00)01849-0]

The improvements in achievable power levels in highpower-microwave devices over the past decade have often been met with a reduction in the corresponding microwavepulse length. ${ }^{1,2}$ This phenomenon is known as microwavepulse shortening and generally holds the radiated energy at more or less a constant as power is increased. Previous experiments ${ }^{1-5}$ have related microwave-pulse shortening to the formation of plasma from the cathode, anode, interaction regions, and e-beam collector of the microwave tube. Our earlier experiments also correlated this plasma with hydrogen alpha-line emission, suggesting that water vapor attached to the inside surfaces of the vacuum system was the major contaminant. ${ }^{4-6}$ Furthermore, experiments at Sandia National Laboratory ${ }^{7,8}$ and the University of Michigan ${ }^{6}$ demonstrated that radio-frequency (rf) plasma cleaning is an effective means of cleaning contaminants from ion- and electron-beam diodes in $\mathrm{MeV}$-pulsed power accelerators.

The purpose of this letter is to demonstrate that rf plasma cleaning can mitigate the effects of microwave-pulse shortening due to plasma formation in pulsed-power, multi-MW microwave sources. The achieved microwave-pulse lengthening is believed to be due to a reduction in the inventory of wall contaminants, primarily water vapor, released during high-power electron-beam collection and microwave production. The subsequent reduction of plasma density growth delays the onset of plasma cutoff of the microwave power, which occurs when the plasma electron density exceeds the critical value:

$$
n=\omega^{2} m \varepsilon_{0} / e^{2},
$$

where $\omega$ is the radian frequency of the microwaves, $e$ and $m$ are the electron charge and mass, and $\varepsilon_{0}$ is the free-space

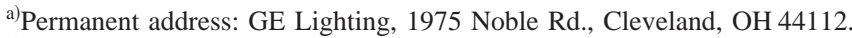

b) Electronic mail: rongilg@umich.edu
}

permittivity. For the operating frequency of these experiments, $2.5 \mathrm{GHz}$, this cutoff electron density is a moderate value of $7.7 \times 10^{10} \mathrm{~cm}^{-3}$.

The experimental configuration is depicted in Fig. 1. The coaxial gyrotron and accelerator have been described in detail elsewhere ${ }^{9,10}$ and only essential features will be given here. The coaxial gyrotron consists of a cusp electron gun, which generates a large-orbit rotating electron beam of energy $0.8 \mathrm{MeV}$, tube current of 300-1000 A, and pulse length of $0.5-0.6 \mu \mathrm{s}$. The electron beam passes through a coaxial cavity followed by a collector in which electrons are dumped. Microwaves with peak power levels of 10-40 MW pass through the collector and exit the device through a Lucite window.

Radio-frequency plasma cleaning is performed by applying to the center coaxial rod about $50 \mathrm{~W}$ of power at a frequency of $13.56 \mathrm{MHz}$. The fill gas is nitrogen at pressures of 15-25 mTorr. When the rf discharge is established, ions accelerated in the rf plasma strike water layers on the surface of the microwave cavity and collector structure and desorb

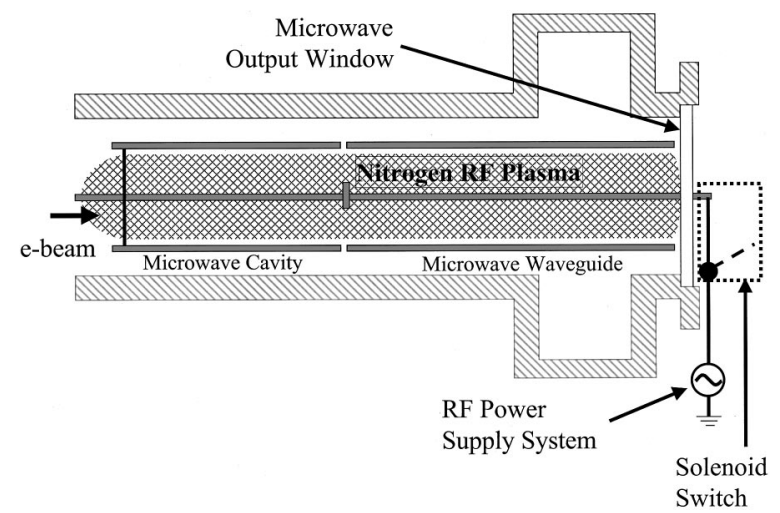

FIG. 1. Cutaway, side view of microwave interaction region. Connection of rf power supply to the coaxial gyrotron center conductor is shown. 


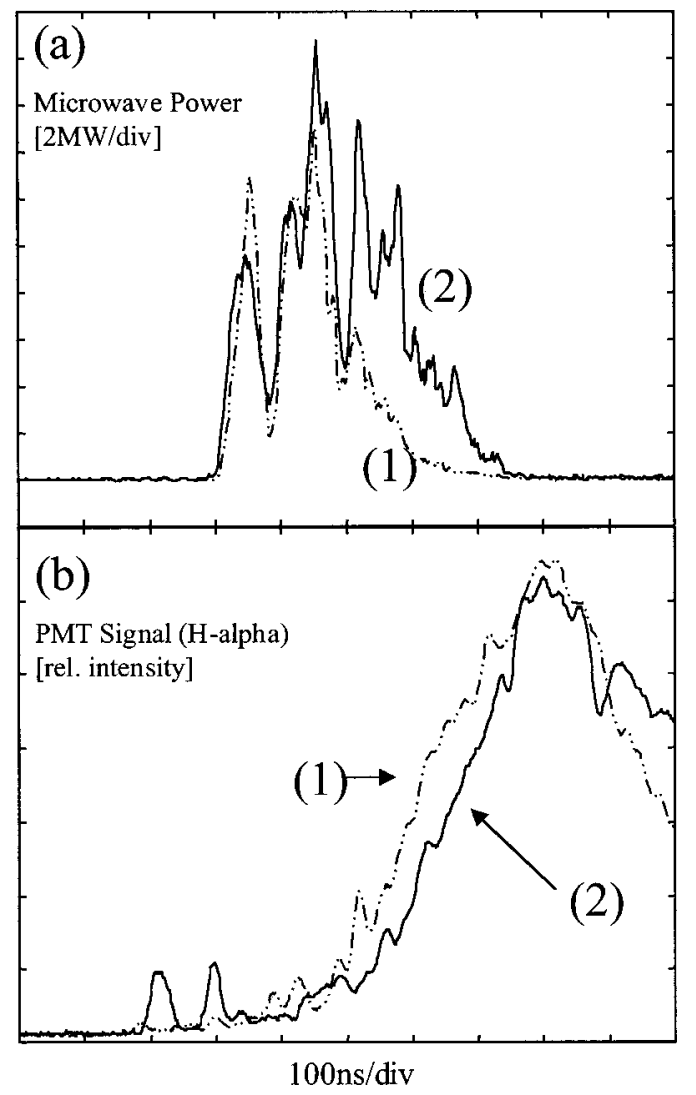

FIG. 2. Digitally averaged (a) microwave power over 15 shots and (b) $\mathrm{H}$-alpha optical emission over 12 shots for uncleaned case (1) and rf cleaned case (2).

water molecules. These desorbed molecules and the fill gas are subsequently removed from the system by two cryogenic pumps. Essentially, the rf plasma cleaning reduces the water inventory available to be released during e-beam operation of the coaxial gyrotron.

Experimental data are presented in Figs. 2 and 3. These microwave emission data were collected to compare two cases:

(1) baseline, uncleaned microwave tube vacuum of $1.3 \times 10^{-5}$ Torr, and

(2) after plasma cleaning by $50 \mathrm{~W}$ for $1.5 \mathrm{~h}$ at a pressure of 25 mTorr nitrogen. Post-rf cleaning vacuum was $1 \times 10^{-5}-7.4 \times 10^{-6}$ Torr.

Figure 2 depicts the digitally averaged microwave-power signals measured by a crystal (diode) detector for cases (1) and (2). For both cases, the first two spikes of microwave power are caused primarily by reproducible e-beam voltage fluctuations. In uncleaned case (1) of Fig. 2(a), it can be seen that the averaged microwave signal from 15 shots is attenuated after the second major spike of power. However, case (2) of plasma cleaning, in Fig. 2(a) (the average of 12 shots), shows that high-power microwaves are emitted for an additional $200 \mathrm{~ns}$ longer than the base vacuum. Figure 2(b) shows the plasma optical emission from the H-alpha line. Radio-frequency plasma cleaning apparently delays the optical emission from this plasma line radiation, indicating a reduction in the initial abundance of water available as a source of $\mathrm{H}$ plasma.
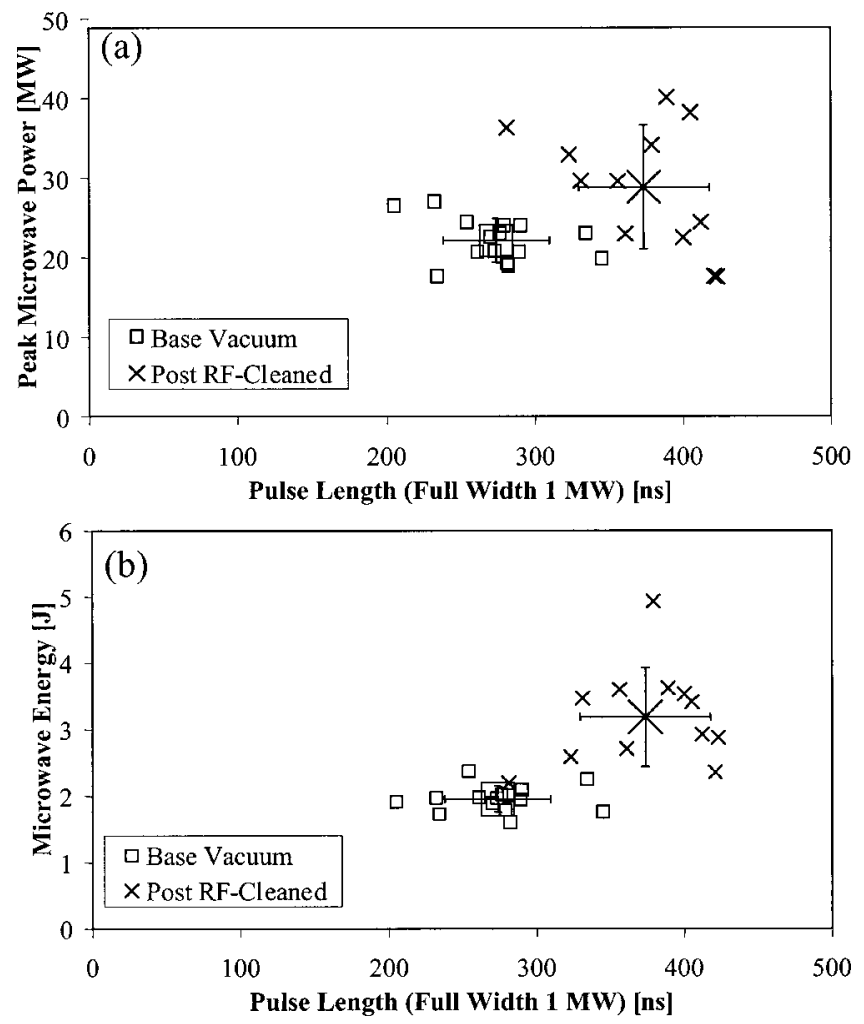

FIG. 3. (a) Peak microwave power and (b) microwave energy plotted vs microwave-pulse length (full width at $1 \mathrm{MW}$ ). The average for each case is represented by an enlarged plot symbol. The standard deviation of this average is given by error bars.

Figure 3 presents a statistical analysis of the microwave emission data for cases (1) and (2). In Fig. 3(a), one can observe an increase of $30 \%$ in the average of the peak microwave power; another observation from these data is an increase of $36 \%$ in the average microwave pulse length. By digitally integrating the individual microwave signals, the per-pulse microwave energy can be calculated and graphed, as shown in Fig. 3(b). The average microwave energy for the plasma cleaning case is increased by $63 \%$ compared to the base vacuum. Utilizing analysis of variance, ${ }^{11}$ these increases in average power, pulse length, and energy exhibit statistical confidence levels above $99 \%$.

Other rf plasma cleaning protocols have been applied, including alternating cleaned and uncleaned shots. However, the post-rf cleaning data exhibited the most statistically significant improvements, ranging up to $245 \%$ energy increase over an extremely contaminated case right after opening the vacuum to atmosphere.

In summary, these data prove that radio-frequency plasma cleaning can exhibit a statistically significant improvement in the average microwave power, pulse length, and energy for the typical, moderate vacuums $\left(10^{-5}-10^{-6}\right.$ Torr $)$ of microwave tubes driven by pulsedpower machines. This research reduces the effect of one of the major mechanisms of microwave-pulse shortening. Other mechanisms are described elsewhere including e-beam voltage fluctuations ${ }^{12}$ and mode competition. ${ }^{9}$

The authors gratefully acknowledge the support of the Air Force Office of Scientific Research and its High Energy Microwave MURI, administered through Texas Tech Uni- 
versity, The Air Force Research Laboratory, and Northrop Grumman Corporation. The authors appreciate the plasma cleaning assistance, advice, support, and encouragement of Josh Rintamaki, Mike Cuneo, Tom Mehlhorn, and Peter Menge.

${ }^{1}$ F. J. Agee, IEEE Trans. Plasma Sci. 26, 235 (1998).

${ }^{2}$ J. Benford and G. Benford, IEEE Trans. Plasma Sci. 25, 311 (1997).

${ }^{3}$ F. Hegeler, C. Grabowski, and E. Schamiloglu, IEEE Trans. Plasma Sci. 26, 275 (1998).

${ }^{4}$ W. E. Cohen, Ph.D. thesis, University of Michigan (2000).

${ }^{5}$ R. M. Gilgenbach, J. M. Hochman, R. L. Jaynes, W. E. Cohen, J. I. Rintamaki, C. W. Peters, D. E. Vollers, Y. Y. Lau, and T. A. Spencer, IEEE Trans. Plasma Sci. 26, 282 (1998).

${ }^{6}$ J. I. Rintamaki, R. M. Gilgenbach, W. E. Cohen, R. L. Jaynes, M. E. Cuneo, and P. R. Menge, Appl. Phys. Lett. 75, 31 (1999).
${ }^{7}$ M. E. Cuneo, P. R. Menge, D. L. Hanson, W. E. Fowler, M. A. Bernard,G. R. Ziska, A. B. Filuk, J. E. Bailey, M. P. Desjarlais, T. R. Lockner, T. A. Mehlhorn, T. D. Pointon, S. A. Slutz, M. A. Stark, and R. A. Vesey, IEEE Trans. Plasma Sci. 25, 229 (1997).

${ }^{8}$ P. R. Menge and M. E. Cuneo, IEEE Trans. Plasma Sci. 25, 252 (1997). ${ }^{9}$ R. L. Jaynes, R. M. Gilgenbach, J. M. Hochman, N. Eidietis, J. I. Rintamaki, W. E. Cohen, C. W. Peters, Y. Y. Lau, and T. A. Spencer, IEEE Trans. Plasma Sci. 27, 136 (1999).

${ }^{10}$ R. L. Jaynes, R. M. Gilgenbach, C. W. Peters, W. E. Cohen, M. R. Lopez, Y. Y. Lau, W. J. Williams, and Thomas A. Spencer, IEEE Trans. Plasma Sci. (to be published).

${ }^{11}$ R. J. Freund and W. J. Wilson, Statistical Methods, revised edition (Academic, New York, 1997).

${ }^{12}$ C. W. Peters, R. L. Jaynes, Y. Y. Lau, R. M. Gilgenbach, W. J. Williams, J. M. Hochman, W. E. Cohen, J. I. Rintamaki, D. E. Vollers, and T. A. Spencer, Phys. Rev. E 58, 6880 (1998). 